\title{
Magnetotunneling of holes through single and double barriers using a multiband treatment
}

\author{
P. Krstajić* and F. M. Peeters ${ }^{\dagger}$ \\ Departement Fysica, Universiteit Antwerpen (Campus Drie Eiken), Universiteitsplein 1, B-2610 Antwerpen, Belgium
}

(Received 25 June 2004; revised manuscript received 16 November 2004; published 23 March 2005)

\begin{abstract}
We investigate tunneling of holes through a single barrier which is subject to an in-plane magnetic field. Band mixing between heavy and light holes is pronounced which indicates the necessity of using a multiband approach. The problem is investigated within the $4 \times 4$ Luttinger-Kohn model for bands at $\Gamma_{8}$, without restriction on the Luttinger parameters. The application of a magnetic field enhances the anisotropy in the transmission coefficients $T_{\lambda \mu}\left(k_{x}, k_{y}\right)$ and makes transitions possible between various channels (e.g., the two light holes). It is shown that heavy holes can precess when magnetic field is in plane, since band mixing lifts the selection rules for angular momentum. The current density is calculated for a double-barrier resonant structure and compared with a previous approximate theoretical result. This comparison clearly indicates that full wave vector dependence of the tunneling process is needed in order to obtain reliable values for the current.
\end{abstract}

DOI: 10.1103/PhysRevB.71.115321

PACS number(s): 73.40.Kp, 75.50.Pp, 73.50.Jt

\section{INTRODUCTION}

The discovery ${ }^{1}$ of diluted magnetic semiconductors (DMS's) has reinforced interest in spin-dependent phenomena in semiconductor heterostructures, especially those made $^{2,3}$ of $(\mathrm{GaMn}) \mathrm{As}$ and AlAs. Since $(\mathrm{GaMn})$ As is a $p$-type material, with holes being majority carriers, a multiband theory that takes into account the degeneracy at $\Gamma_{8}$ is needed. First, the hole tunneling in nonmagnetic GaAs/AlAs in a magnetic field should be first investigated and clarified. One of the simplest and most widely used models is the $4 \times 4$ Luttinger Kohn k·p Hamiltonian, ${ }^{4,5}$ which includes explicitly only heavy and light holes at the $\Gamma_{8}$ point. Though more accurate methods such as the effective bond-orbital method (EBOM) were recently employed to investigate intraband ${ }^{6}$ tunneling, the $\mathbf{k} \cdot \mathbf{p}$ theory provides a more clear physical insight into band mixing. It is known that it can predict the positions of resonant peaks in double-barrier heterostructures ${ }^{7}$ with reasonable accuracy and explain the difference between the tunneling lifetimes of heavy and light holes. $^{8}$

In this paper the tunneling through a single barrier is examined in detail with and without a magnetic field applied to the barrier. The problem is treated numerically by solving the coupled system of differential equations as proposed by Xia. ${ }^{9}$ Although recently the multiband quantum transmitting boundary method ${ }^{10}$ (MQTBM) was developed to treat this kind of problems, we find that the method used here requires less processor time when the mesh size is made arbitrary small. It surpasses the transfer matrix method (TMM) in numerical stability when the layer widths ${ }^{11}$ exceed $40-50 \AA$, due to the numerical difficulty in dealing with numbers with high discrepancy in order of magnitude that arises in the TMM. Magnetotunneling in GaAs-AlGaAs has already been treated in several works, ${ }^{12,13}$ but either with the neglect of the material dependence of the Luttinger parameters or within the spherical approximation $\left(\gamma_{2}=\gamma_{3}\right)$ or in the absence of a magnetic field. ${ }^{11,14,15}$ Here, we will go beyond these approximations.

The paper is organized as follows. In Sec. II the $4 \times 4$ Luttinger-Kohn (LK) model is reexamined, with particular attention paid to its application to the tunneling problem and in the most general case when all the Luttinger parameters are different. In Sec. III, the method for numerical calculations is explained. In Sec. IV we give results for the transmission and reflection coefficients as a function of in-plane momenta, in the absence and presence of a magnetic field. In the subsequent section the tunneling current in a resonant tunneling device is given for $\mathbf{B}=0$ and in the case of a magnetic field in plane, $\mathbf{B} \| x$, which is more interesting for practical applications. A summary of the results is presented in Sec. VI.

\section{MODEL}

The basic idea of the effective mass theory is that the motion of an electron or a hole can be described by the effective mass tensor (in general) if the external potential $V$ varies smoothly through the unit cell and the Bloch functions at the top of the bands do not change rapidly across different materials in the heterostructure. In the case of both GaAs and AlAs the split-off (SO) band lies sufficiently below the $\Gamma_{8}$ point and the $4 \times 4$ LK Hamiltonian is sufficiently accurate to explain the behavior of the holes (at least in the vicinity of $\mathbf{k}=\mathbf{0})$ and reads 9

$$
H=\left(\begin{array}{cccc}
P_{1} & Q & R & 0 \\
Q^{\dagger} & P_{2} & 0 & R \\
R^{\dagger} & 0 & P_{2} & -Q \\
0 & R^{\dagger} & -Q^{\dagger} & P_{1}
\end{array}\right)+V(z) I_{4}-2 \kappa \mu_{B} \mathbf{J} \cdot \mathbf{B}
$$

where

$$
\begin{aligned}
& P_{1}=\frac{\hbar^{2}}{2 m_{0}}\left[\left(\gamma_{1}+\gamma_{2}\right)\left(k_{x}^{2}+k_{y}^{2}\right)+\left(\gamma_{1}-2 \gamma_{2}\right) k_{z}^{2}\right], \\
& P_{2}=\frac{\hbar^{2}}{2 m_{0}}\left[\left(\gamma_{1}-\gamma_{2}\right)\left(k_{x}^{2}+k_{y}^{2}\right)+\left(\gamma_{1}+2 \gamma_{2}\right) k_{z}^{2}\right],
\end{aligned}
$$




$$
\begin{gathered}
Q=-i \frac{\sqrt{3} \hbar^{2}}{m_{0}} \gamma_{3}\left(k_{x}-i k_{y}\right) k_{z}, \\
R=\frac{\sqrt{3} \hbar^{2}}{2 m_{0}}\left[\gamma_{2}\left(k_{x}^{2}-k_{y}^{2}\right)-2 i \gamma_{3} k_{x} k_{y}\right] .
\end{gathered}
$$

Here $\gamma_{i}$ are the well-known Luttinger parameters (extracted from experiment) and $\kappa$ is an additional phenomenological parameter describing the coupling of the hole with an external magnetic field. The basis eigenfunctions $\{|j, m\rangle\}(j$ $=3 / 2)$ are ordered as $\{|3 / 2,3 / 2\rangle,|3 / 2,1 / 2\rangle, \mid 3 / 2$, $-1 / 2\rangle,|3 / 2,-3 / 2\rangle\}$ and represent the periodic part of the Bloch functions at $\mathbf{k}=\mathbf{0}\left(\Gamma_{8}\right)$. They correspond to the states of heavy holes $h_{1}$ and $h_{2}(m= \pm 3 / 2)$ and light holes $l_{1} l_{2}$ $(m= \pm 1 / 2)$. The axis of quantization of the angular momentum $\mathbf{J}$ is chosen to be along the growth direction ${ }^{12,16}$ _for instance, $z$-and hence $k_{z} \rightarrow \hat{k}_{z}=-i \partial_{z}$. The off-diagonal terms $Q$ and $R$ cause the mixing of heavy and light holes for finite in-plane momentum $k_{x}, k_{y}$.

In the presence of a magnetic field, the wave vectors $k_{i}$ must be replaced by $k_{i}-e A_{i} / \hbar, i=x, y, z$, and they no longer commute. It was already noticed in the beginning of the development of the theory ${ }^{4}$ that in order to preserve the Hermicity of the Hamiltonian a symmetrized combination of the wave vectors $k_{i} k_{j} \rightarrow 1 / 2\left(k_{i} k_{j}+k_{j} k_{i}\right)$ must be used in the matrix elements in Eq. (2). Now, in order to rewrite the Hamiltonian in a form suitable for numerical calculations one should normally move the operator $\hat{k}_{z}=-i \partial_{z}$ to the rightmost place, ${ }^{13}$ which leads to an extra term in the Hamiltonian:

$$
H=H_{0}+\left(\begin{array}{cccc}
0 & K & 0 & 0 \\
K & 0 & 0 & 0 \\
0 & 0 & 0 & -K \\
0 & 0 & -K & 0
\end{array}\right) \text {, }
$$

where $H_{0}$ is the LK Hamiltonian in which $\hat{k}_{z}$ stands always at the rightmost end in the off-diagonal terms $\pm Q, \pm Q^{\dagger}$ (and thus using complex conjugation instead of Hermitian conjugation). The term $K$ is equal to $1 / 2\left[\hat{k}_{z}, Q_{1}\right]$, where $Q_{1}$ is the factor in $Q$ [Eq. (2c)] in the front of $\hat{k}_{z}$. We assume that the external leads of the structure are not affected by the magnetic field (only by the vector potential A) and the solution of the Schrödinger equation $H \Psi=E \Psi$ in the bulk where $\mathbf{B}=\mathbf{0}$ is a linear combination of the plane waves

$$
\Psi(\mathbf{r})=e^{i\left(k_{x} x+k_{y} y\right)} \sum_{i=1,8} a_{i}^{(L, R)}\left(\begin{array}{c}
F_{1} \\
F_{2} \\
F_{3} \\
F_{4}
\end{array}\right) e^{i k_{z i} z} .
$$

Here $\left(F_{1}, F_{2}, F_{3}, F_{4}\right)^{T}=V_{i}$ represents the four-column eigenvectors (see, e.g., Ref. 17) of the Hamiltonian at the same energy $E$ and the wave vector $k_{z}$ classifies the states of heavy and light holes. The eigenvectors $V_{i}$ should be normalized to unity to ensure that the coefficients $a_{i}^{(L, R)}$ are directly proportional to the probability current density of the particular channels-i.e., to transmission (reflection) coefficients.
This is particularly important if there is an applied bias across the structure, when even transmission of holes of the same kind must be normalized by the group velocity. In so doing, care must be taken during evaluation of the Hermitian conjugate of an eigenvector $V_{i}^{\dagger}=\left(F_{1}^{*}, F_{2}^{*}, F_{3}^{*}, F_{4}^{*}\right)$ that whenever a term contains $k_{z}$, it must be kept unchanged ${ }^{17}$-i.e., $\left\{F_{k}\left(k_{z}\right)\right\}^{*}=F_{k}^{*}\left(k_{z}\right)$-even when it is a complex number. This is the consequence of the fact that the corresponding operator $-i \partial_{z}$ is Hermitian and the value of the wave vector $k_{z}$ is determined by the biquadratic equation

$$
\boldsymbol{\epsilon}=\gamma_{1} k^{2} \pm \sqrt{4 \gamma_{2}^{2} k^{4}+C^{2}\left(k_{x}^{2} k_{y}^{2}+k_{y}^{2} k_{z}^{2}+k_{z}^{2} k_{x}^{2}\right)},
$$

where $\epsilon=2 m_{0}(E-V) / \hbar^{2}$ is the normalized energy in the flat potential regions and $C^{2}=12\left(\gamma_{3}^{2}-\gamma_{2}^{2}\right)$. The plus (minus) sign corresponds to light (heavy) holes. Depending on the energy and values of the in-plane momenta $k_{x}, k_{y}$, the solution of Eq. (5) with respect to $k_{z}$ can have the following properties:

(i) $\epsilon>\epsilon_{c 4}$, all $k_{z h}$ and $k_{z l}$ are real,

(ii) $\epsilon_{c 4}>\epsilon>\epsilon_{c 3}, k_{z h}$ is real, and $k_{z l}$ purely imaginary,

(iii) $\epsilon_{c 3}>\epsilon>\epsilon_{c 2}, k_{z h}$ and $k_{z l}$ are real,

(iv) $\epsilon_{c 2}>\epsilon>\epsilon_{c 1}, k_{z h}$ and $k_{z l}$ are complex,

(v) $\epsilon<\epsilon_{c 1}$, all $k_{z h}$ and $k_{z l}$ are purely imaginary,

where

$$
\begin{aligned}
\boldsymbol{\epsilon}_{c 1}= & \frac{1}{2 \gamma_{2}^{2}}\left(-3 \gamma_{1}\left(\gamma_{3}^{2}-\gamma_{2}^{2}\right) k_{t}^{2}-\left\{3\left(\gamma_{1}^{2}-4 \gamma_{2}^{2}\right)\left(\gamma_{3}^{2}-\gamma_{2}^{2}\right)\right.\right. \\
& \left.\left.\times\left[\left(3 \gamma_{3}^{2}+\gamma_{2}^{2}\right) k_{t}^{4}-4 \gamma_{2}^{2} k_{x}^{2} k_{y}^{2}\right]\right\}{ }^{1 / 2}\right),
\end{aligned}
$$

$$
\begin{gathered}
\epsilon_{c 2}=\frac{1}{2 \gamma_{2}^{2}}\left(-3 \gamma_{1}\left(\gamma_{3}^{2}-\gamma_{2}^{2}\right) k_{t}^{2}+\left\{3\left(\gamma_{1}^{2}-4 \gamma_{2}^{2}\right)\left(\gamma_{3}^{2}-\gamma_{2}^{2}\right)\right.\right. \\
\left.\left.\times\left[\left(3 \gamma_{3}^{2}+\gamma_{2}^{2}\right) k_{t}^{4}-4 \gamma_{2}^{2} k_{x}^{2} k_{y}^{2}\right]\right\}^{1 / 2}\right) \\
\epsilon_{c 3}=\gamma_{1} k_{t}^{2}-\left[4 \gamma_{2}^{2} k_{t}^{4}+12\left(\gamma_{3}^{2}-\gamma_{2}^{2}\right) k_{x}^{2} k_{y}^{2}\right]^{1 / 2} \\
\epsilon_{c 4}=\gamma_{1} k_{t}^{2}+\left[4 \gamma_{2}^{2} k_{t}^{4}+12\left(\gamma_{3}^{2}-\gamma_{2}^{2}\right) k_{x}^{2} k_{y}^{2}\right]^{1 / 2}, \quad k_{t}^{2}=k_{x}^{2}+k_{y}^{2}
\end{gathered}
$$

The most interesting case is (iii) when all (real) solutions lie on the heavy-hole branch (see Fig. 1). Due to its warped structure, the nominally classified light-hole states (with smaller $k_{z}$ ) have group velocity with opposite sign than that of its phase velocity. It is important to bear this in mind during the calculation of the transmitivity $T$ and reflectivity $R$ coefficients, because they are normalized by the probability density current-i.e., by the group velocity:

$$
\begin{aligned}
& T_{\lambda \mu}=\frac{\left|a_{\mu}^{(R)}\right|^{2}}{\left|a_{\lambda}^{(L)}\right|^{2}} \frac{j_{z 0 \mu}^{(R)}}{j_{z 0 \lambda}^{(L)}}=\left|t_{\lambda \mu}^{0}\right|^{2} \frac{v_{z \mu}^{(R)}}{v_{z \lambda}^{(L)}}, \\
& R_{\lambda \mu}=\frac{\left|a_{\mu}^{(L)}\right|^{2}}{\left|a_{\lambda}^{(L)}\right|^{2}} \frac{j_{z 0 \mu}^{(L)}}{j_{z 0 \lambda}^{(L)}}=\left|r_{\lambda \mu}^{0}\right|^{2} \frac{v_{z \mu}^{(L)}}{v_{z \lambda}^{(L)}} .
\end{aligned}
$$

Here 


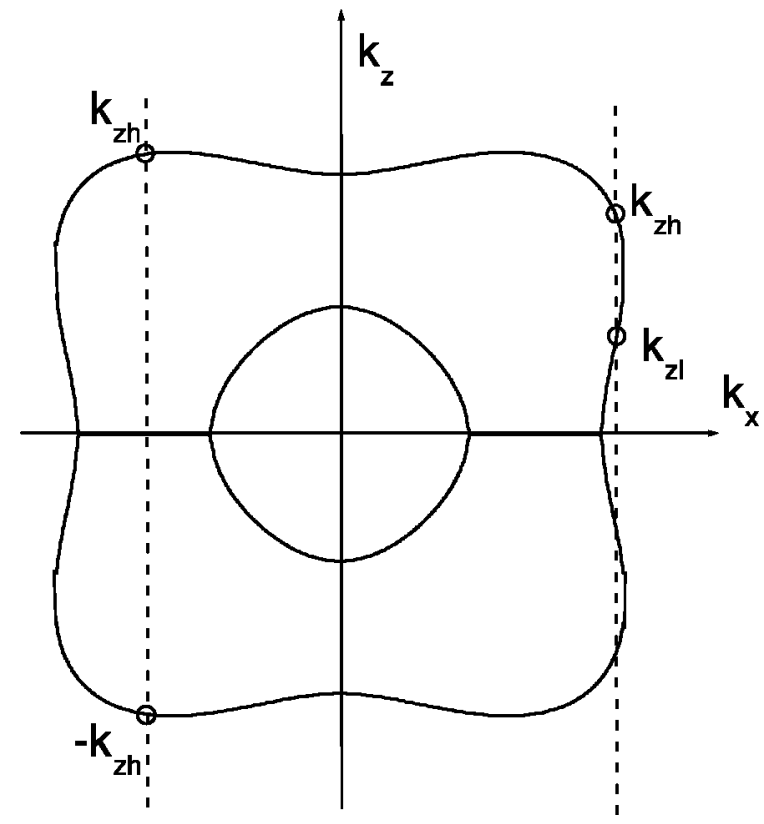

FIG. 1. Constant-energy diagram for light and heavy holes determined by Eq. (5), at $k_{y}=0$. For certain $k_{x}$ (right vertical dashed line) all solutions lie on the heavy-hole branch (the outer contour), the case (iii), with light holes having negative slope $\nabla_{\mathbf{k}} E(\mathbf{k})$. The left vertical dashed line shows case (ii) in the text, where the heavy hole propagates, while the light holes have evanescent wave functions.

$$
v_{z h, l}=\frac{\hbar k_{z h, l}}{m_{0}} \frac{\left(\gamma_{1}^{2}-4 \gamma_{2}^{2}\right) k^{2}-6\left(\gamma_{3}^{2}-\gamma_{2}^{2}\right) k_{t}^{2}-\gamma_{1} \epsilon}{\gamma_{1} k^{2}-\epsilon}
$$

is the group velocity of both heavy and light holes, $v_{z}$ $=\hbar^{-1} \partial_{z} E$. It was proved ${ }^{18}$ that it is directly proportional to the probability current density even in case of degenerate bands $\mathbf{j}=\rho \mathbf{v}(\mathbf{k})$, which justifies using $v_{z}$ instead of $j_{z 0}$ in Eqs. (7), as $\rho_{0}=V_{i}^{\dagger} V_{i}=1$. Furthermore, since the LK Hamiltonian describes a hole in four possible states (the two light- and the two heavy-hole states), there are four channels possible, and due to band mixing, for instance, a heavy hole passing through the barrier can end up as either a heavy hole or a light hole with certain probabilities determined by the Schrödinger equation (see Fig. 2). The difference in the velocities of light and heavy holes outside the barrier must be accounted for, during this conversion, as was already done in Eq. (9). Therefore, the conservation of the probability current $\operatorname{div} \mathbf{j}=0$ leads to four relations among the transmission and reflection coefficients:

$$
R_{\lambda h_{1}}+R_{\lambda h_{2}}+R_{\lambda l_{1}}+R_{\lambda l_{2}}+T_{\lambda h_{1}}+T_{\lambda h_{2}}+T_{\lambda l_{1}}+T_{\lambda l_{2}}=1 .
$$

Here the variable $\lambda$ assumes all four states $\left(h_{1}, h_{2}, l_{1}, l_{2}\right)$. These relations are also useful to check the consistency of the numerical calculations. However, for certain values of the in-plane momentum $k_{x}, k_{y}$ the wave vector $k_{z l}$ of the light hole is purely imaginary [case (ii); see Fig. 1]; i.e., their wave functions are evanescent. The quantity $\operatorname{Re}\left(\Psi^{*} \hat{j}_{z} \Psi\right)$ can no longer be related to the particle flux, and one should put

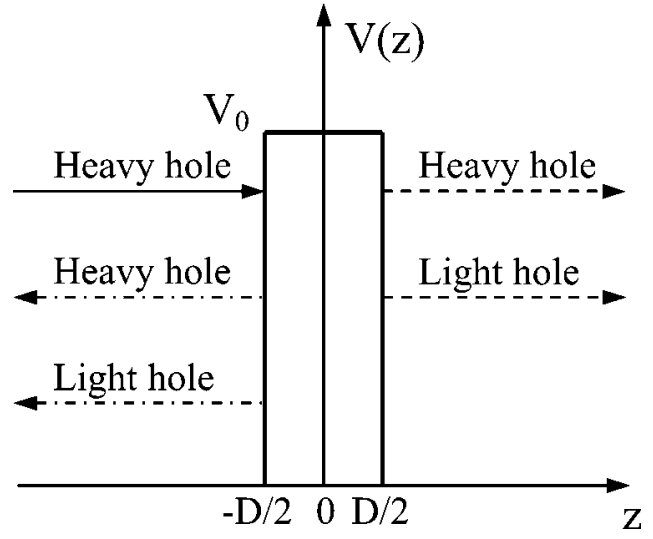

FIG. 2. A heavy hole impinging on a barrier can transmit (reflect) as a heavy hole or as a light hole on the right (left) side due to band mixing in the barrier (similarly for a light hole).

by hand $T_{\lambda l}=0$ and $T_{l \mu}=0$ (for both light holes $l=l_{1}, l_{2}$ ), as well as for the corresponding power reflection coefficients. Therefore the conservation relations, Eq. (9), reduce to only two:

$$
R_{\lambda h_{1}}+R_{\lambda h_{2}}+T_{\lambda h_{1}}+T_{\lambda h_{2}}=1, \quad \lambda \in\left\{h_{1}, h_{2}\right\} .
$$

However, it may happen that under a positive bias and for some $\left(k_{x}, k_{y}\right)$, the light holes have propagating states only in the right lead, so that Eq. (9) turns into

$$
R_{\lambda h_{1}}+R_{\lambda h_{2}}+T_{\lambda h_{1}}+T_{\lambda h_{2}}+T_{\lambda l_{1}}+T_{\lambda l_{2}}=1
$$

for $\lambda \in\left\{h_{1}, h_{2}\right\}$.

\section{NUMERICAL METHOD}

The major difference between tunneling phenomena and, e.g., treatment of bound states in semiconductor physics, from the numerical point of view, is that the boundary conditions are not known in advance in the former case. This is due to the presence of reflected waves, whose amplitude is yet to be found, and the traditional method to tackle it has been the transfer matrix method as developed earlier to treat analog problems in classical optics. An alternate way was developed by Frensley ${ }^{19}$ for electrons and then later by $\mathrm{Yu}$ and co-workers ${ }^{8}$ for holes (i.e., for the case of degenerate bands, now called MQTBM) where the problem is solved by a method similar as in a finite-difference scheme but here adapted for the propagating states. The basic idea of the MQTBM is to add an extra layer in the left lead, still in the flatband region, in order to eliminate the unknown reflection coefficients. However, we treat the problem as a set of coupled differential equations, which is solved by the Adams method $^{20}$ (it belongs to the class of predictor-corrector methods). The procedure was originally outlined and explained in Ref. 9 and it will be repeated briefly here. It exploits the superposition principle in the solution of the Schrödinger equation. Let us define the matrix $M$ that connects output and input channels-i.e., the coefficients of expansion in Eq. (4): 


$$
\left(\begin{array}{c}
a_{h 1} \\
a_{h 2} \\
a_{l 1} \\
a_{l 2} \\
r_{h 1} \\
r_{h 2} \\
r_{l 1} \\
r_{l 2}
\end{array}\right)=M\left(\begin{array}{c}
t_{h 1} \\
t_{h 2} \\
t_{l 1} \\
t_{l 2} \\
0 \\
0 \\
0 \\
0
\end{array}\right) .
$$

Note that the matrix $M$ connects the coefficients from the right flatband region to the left, in the opposite direction than that in the TMM. Now if we put $t_{\lambda}=1$ for some $\lambda$ $\in\left(h_{1}, h_{2}, l_{1}, l_{2}\right)$ and take the others zero (on the right lead), then we know the boundary conditions on the right side of the barrier, since there are no reflected waves. These are sufficient to solve the set of coupled equations arising from the $4 \times 4$ LK Hamiltonian, going backward to the left lead by discretizing the structure by a mesh with step $h=\Delta z=D / N$, where $D$ is the barrier width. Then the solution, obtained by numerical integration, is decomposed on the left side as a linear combination, likewise Eq. (4) of normalized eigenfunctions to obtain the coefficients $a_{i}^{L}$. These are actually the entries or, more precisely, $\lambda$ th column of the matrix $M$, since $t_{\lambda}=1$. Upon repeating the whole procedure for each four outgoing states, one finds all entries in the left half of the matrix, which we denote by two $4 \times 4$ submatrices $M^{U}, M^{L}$ :

$$
M=\left(\begin{array}{ll}
M^{U} & * \\
M^{L} & *
\end{array}\right) .
$$

The right half of $M$ is not important due to the absence of reflected waves on the right side of the barrier-i.e., due to zeros in the outgoing vector in Eq. (12). It can be easily proved that

$$
T_{i j}=\left|N_{j i}\right|^{2} \frac{v_{z j}^{(R)}}{v_{z i}^{(L)}}, \quad R_{i j}=\left|\sum_{k=1,4} N_{j, k} M_{k, i}^{L}\right| \frac{2 v_{z j}^{(L)}}{v_{z i}^{(L)}},
$$

where $i, j \in\{1,2,3,4\}=\left\{h_{1}, h_{2}, l_{1}, l_{2}\right\}, N=\left(M^{U}\right)^{-1}$. The major advantage of the method presented here is that the calculation time increases linearly with the number of mesh points $N$-i.e., when the step $h$ is diminished-in contrast with the MQTBM where it increases as $O\left(N^{2}\right)$, as the order of the matrix equation that has to be solved is $4(N+3)$.

\section{TRANSMISSION AND REFLECTION COEFFICIENTS: SINGLE BARRIER}

\section{A. Zero-magnetic-field case: $B=0$}

The theory will be applied to the case of a barrier made of $\mathrm{Al}_{0.2} \mathrm{Ga}_{0.8} \mathrm{As}$ which is embedded in GaAs. The barrier width is taken to be $D=25 \AA$, the barrier height-i.e., the valenceband mismatch between the two materials-is $V_{0}=95 \mathrm{meV}$, and the Luttinger parameters are $\gamma_{1}^{a}=6.85, \gamma_{2}^{a}=2.1$, and $\gamma_{3}^{a}$ $=2.9(\mathrm{GaAs})$ and $\gamma_{1}^{b}=3.45, \gamma_{2}^{k}=0.68$, and $\gamma_{3}^{2}=1.29(\mathrm{AlAs})$

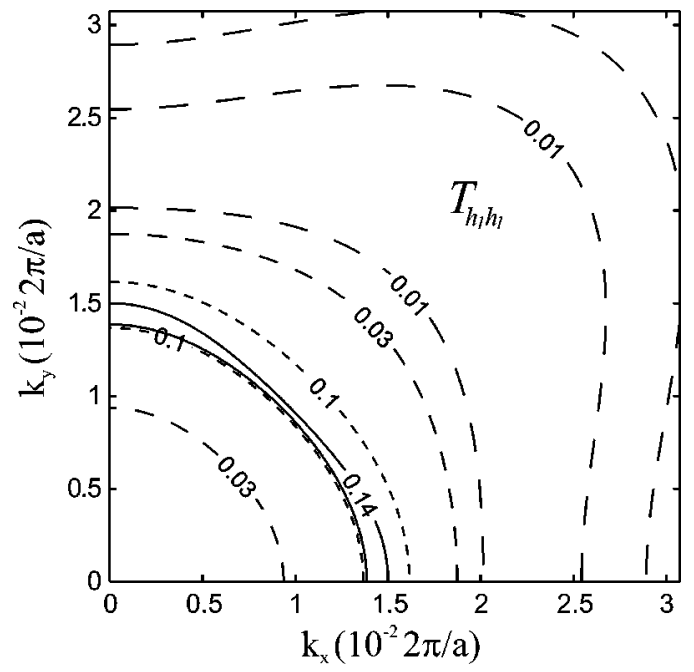

FIG. 3. Contour diagram of the transmission coefficient $T_{h_{1} h_{1}}$ for heavy holes of the same kind with energy $E=10 \mathrm{meV}$, in units of the reciprocal vector $2 \pi / a$. Due to Kramer's degeneracy, the graph is identical to $T_{h_{2} h_{2}}$.

(linear interpolation of $\gamma_{i}$ was used for the alloy $\left.\mathrm{Al}_{x} \mathrm{Ga}_{1-x} \mathrm{As}\right)$. The Fermi energy is taken to be $E_{F}=10 \mathrm{meV}$. In the absence of a magnetic field the wave wectors $k_{x}, k_{y}$, and $k_{z}$ commute with each other and the term $K$ equals zero. The in-plane wave vector $\mathbf{k}_{\|}=\left(k_{x}, k_{y}\right)$ is still a good quantum number when inelastic processes at the interfaces are neglected. The difference in the Luttinger parameters $\gamma_{2} \neq \gamma_{3}$ causes anisotropy in the plane parallel to the interfaces and it will be then important to investigate the dependance $T_{\lambda \mu}$ on the in-plane momentum $\mathbf{k}_{\|}=\left(k_{x}, k_{y}\right)$. A contour plot of the transmission amplitude $T_{h_{1} h_{1}}\left(k_{x}, k_{y}\right)$ between the two heavy holes is shown in Fig. 3. There is a clear anisotropy in the dispersion relation $E\left(k_{x}, k_{y}\right)$ as reflected in this graph. For small $k_{x}$ and $k_{y}$ the contour lines resemble circles as band mixing is less pronounced. However, as the in-plane momentum is increased beyond the first critical value $k_{t}$ $\approx \sqrt{\epsilon /\left(\gamma_{1}+2 \gamma_{2}\right)}$ (when the light holes become evanescent), the contour lines become more distorted. The deviation from isotropic shape is maximal when $k_{x}, k_{y}$ lie in the region (iii) -i.e., when all solutions lie on the heavy-hole branch. The latter is expected since the dispersion relation has now a pronounced warped structure. In this case the term $C^{2} k_{x}^{2} k_{y}^{2}$ becomes dominant in the dispersion relation, when both $k_{x}$ and $k_{y}$ are large, which makes the wave vector $k_{z}$ small and the transmission coefficient $T_{h_{1} h_{1}}$ attains very small values below $10^{-2}$, presented by the dashed lines in the corners of the graph of Fig. 3. Now in order to understand better the contour graph, it is also instructive to have a look at the dependence $T_{h_{1} h_{1}}\left(k_{x}\right)$ at fixed $k_{y}$, which is given in Fig. 4 for three different values of $k_{y} /(2 \pi / a): 0.0$ (solid line) 6 $\times 10^{-3}$ (dashed line), and 0.012 (dash-dotted line). Due to band mixing, the transmission coefficient of the heavy holes increases with $k_{x}$ which is opposite to the predictions of the simple one-band picture. This increase continues as long as the propagating states of the light holes exist, as is so for case (i): the peaks in the graph for different $k_{y}$ indeed correspond to the critical energy $\epsilon_{c 4}$ [Eq. (6d)]. Beyond the maxi- 


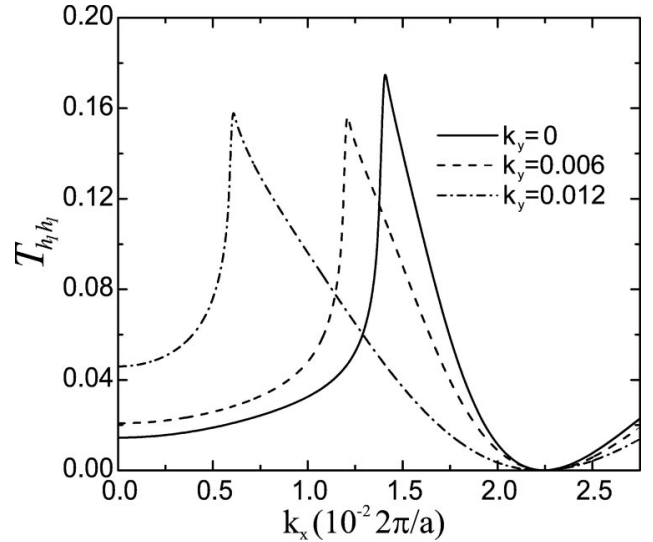

FIG. 4. Transmission coefficient $T_{h_{1} h_{1}}$ for heavy holes as a function of $k_{x}$ for three different values of $k_{y}$ : 0.0 (solid line), 0.006 $\times 2 \pi / a$ (dashed line), and $0.012 \times 2 \pi / a$ (dot-dashed line). The energy is $E=10 \mathrm{meV}$.

mum, it decreases and begins to increase again when values of $\left(k_{x}, k_{y}\right)$ are large enough for the "light holes" to become propagating. It was noticed ${ }^{8,21}$ previously that the heavy-hole tunneling times through double-barrier structures are significantly shorter than can be concluded from their mass $m_{h h}$ $=m_{0} /\left(\gamma_{1}-2 \gamma_{2}\right)$ at $\mathbf{k}_{\|}=0$. Yu et al. ${ }^{8}$ attributed this discrepancy to band mixing in the sense that heavy holes in a barrier acquire somewhat of a light-hole character, having lighter mass and thus enhancing the tunneling of the heavy holes. ${ }^{13}$ Their results were obtained from the $4 \times 4$ LK model, as used in the present paper as well, and are in good agreement with the experimental results of Ref. 21.

Since the heterostructure has inversion symmetry (in the absence of an applied bias), the two heavy-hole states (and the two light-hole states) are degenerate (Kramer's degeneracy) and hence the corresponding coefficients are identical, so that it will suffice to show only $T_{l_{1} l_{1}}\left(=T_{l_{2} l_{2}}\right)$ and $T_{h_{1} l_{1}}$ $\left(=T_{h_{2} l_{2}}\right)$, which are given in Figs. 5(a) and 5(b). The transmission coefficient between the two light holes as a function of $k_{x}, k_{y}$ is more isotropic than that of the heavy holes since their propagating states exist only for relatively small $k_{t}$ $<\sqrt{\epsilon /\left(\gamma_{1}+2 \gamma_{2}\right)}$. The values of $T_{l_{1} l_{1}}$ are larger than that of $T_{h_{1} h_{1}}$, since the light holes transmit through the barrier more easily due to their lighter mass. The transmission coefficients $T_{h_{1} h_{2}}$ and $T_{l_{1} l_{2}}$ vanish identically due to zeros on the side diagonal in the Hamiltonian. We find that the conservation relations (9) and (10) are always fulfilled in contrast to what was found in Ref. 18, even in case (iii), when the light holes propagate in the opposite direction than the phase velocity. In this case one should either assume $T_{h, l}^{\prime}=-R_{h, l}$ and $R_{l, h}^{\prime}$ $=-T_{l, h}$ or choose ${ }^{18}$ a negative value for $k_{z}$ for light holes.

Next we will give the reflection coefficients. There is a difficulty in representing them faithfully on a contour graph since many values are concentrated near unity. However, we choose to present contour lines for 0.01, 0.03, 0.2, and 0.6. The reflection from a heavy to a heavy hole (with the same sign of $m$ ) is given in Fig. 6(a). Total reflection occurs for large values of $\left(k_{x}, k_{y}\right)$ when the propagation wave vector $k_{z}$ is too small to ensure efficient tunneling. Hence, small values of $R_{h_{1} h_{1}}$ are mainly located near the origin $(0,0)$. In the next
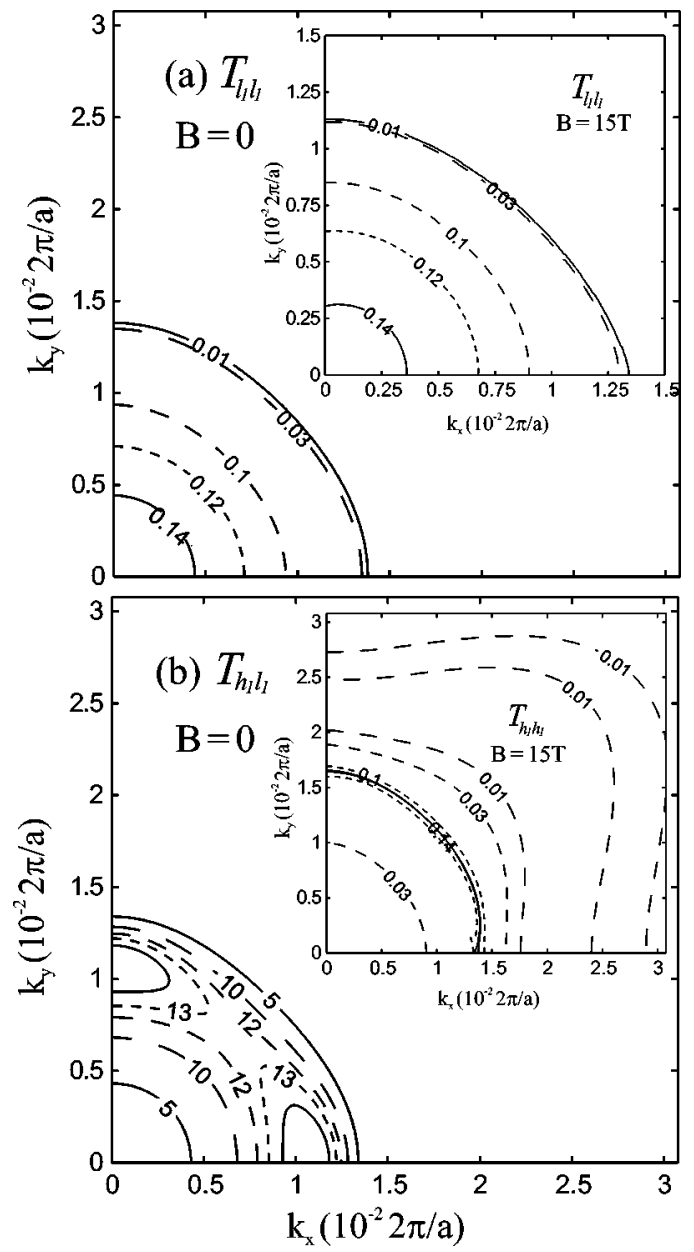

FIG. 5. Contour diagram of the transmission coefficient $T$ : (a) $T_{l_{1} l_{1}}$ for light holes of the same kind in the absence of a magnetic field. Inset: $T_{l_{1} l_{1}}$ for $B=15 \mathrm{~T}$ (b) $T_{h_{1} l_{1}}$ from the heavy to the light holes, at $B=0 \mathrm{~T}$. Inset: $T_{h_{1} h_{1}}$ at $B=0 \mathrm{~T}$. All axes in units of the reciprocal vector $2 \pi / a$, for the energy $E=10 \mathrm{meV}$.

figure, Fig. 6(b), one can see that a heavy hole can reflect as a light hole (but with a negative group velocity) which is denoted by dashed lines in the corners of the graph. For moderate values of the in-plane momentum (around 0.02 $\times 2 \pi / a$ ) there is no reflection as light holes then do not exist. Finally the reflection coefficient as a function of $\left(k_{x}, k_{y}\right)$ from a light hole to a light hole is presented in Fig. 6(c).

\section{B. In-plane magnetic field case: $B \| x$}

When the magnetic field is in plane-i.e., parallel to the interfaces - but acting only in the barrier region-i.e., $z \in(-D / 2, D / 2)$ - and aligned along the $x$ axis, the wave vectors $k_{x}, k_{y}, k_{z}$ no longer commute. For the case of the commonly used Landau gauge $\mathbf{A}=(0,-B z, 0)$ the term $Q$ contains both noncommuting $z$ and $\hat{k}_{z}$, and the term $K$ is equal to $K=i \sqrt{3} \hbar /\left(2 m_{0}\right) \gamma_{3} e B$. The vector potential component $A_{y}$ is constant outside the barrier $A_{y}(z<-D / 2)$ $=+B D / 2, A_{y}(z>+D / 2)=-B D / 2$, and except for the constant shift in the wave vector $k_{y}=k_{y}^{0}-e A_{y} / \hbar=k_{y}^{0}+e B z / \hbar$, the 


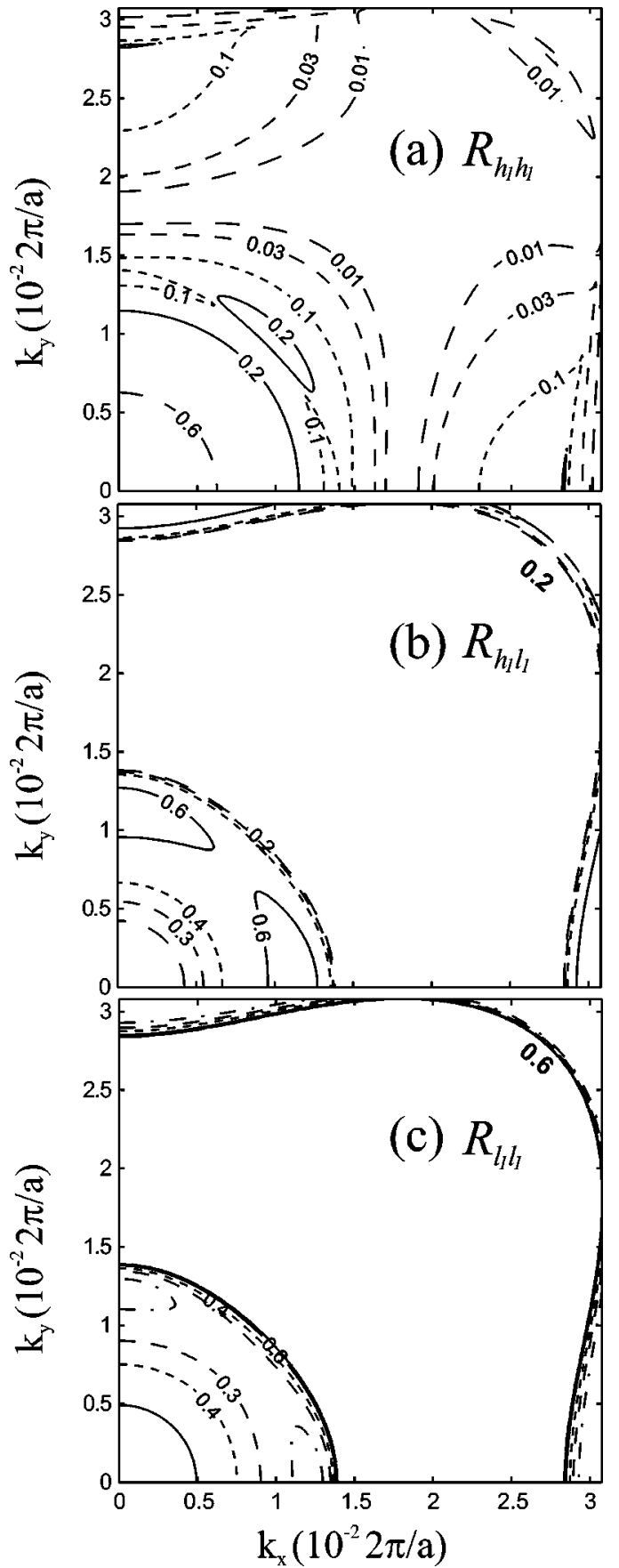

FIG. 6. Contour diagram of reflection coefficients: (a) $R_{h_{1} h_{1}}$ for heavy holes of the same kind (due to Kramer's degeneracy the graph is identical to $R_{h_{2} h_{2}}$ ), (b) $R_{h_{1} l_{1}}$ from the heavy holes to the light holes, and (c) $R_{l_{1} l_{1}}$ from a light hole to a light hole with the same $m=3 / 2$ in units of the reciprocal vector $2 \pi / a$. The energy is $E=10 \mathrm{meV}$.

solution in the external leads is similar to the nonmagnetic case [Eq. (4)]:

$$
\Psi(\mathbf{r})=e^{i\left(k_{x}^{0} x+k_{y}^{0} y\right)} \sum_{i=1,8} a_{i}^{(L, R)}\left(\begin{array}{c}
F_{1} \\
F_{2} \\
F_{3} \\
F_{4}
\end{array}\right) e^{i k_{z i} z} .
$$

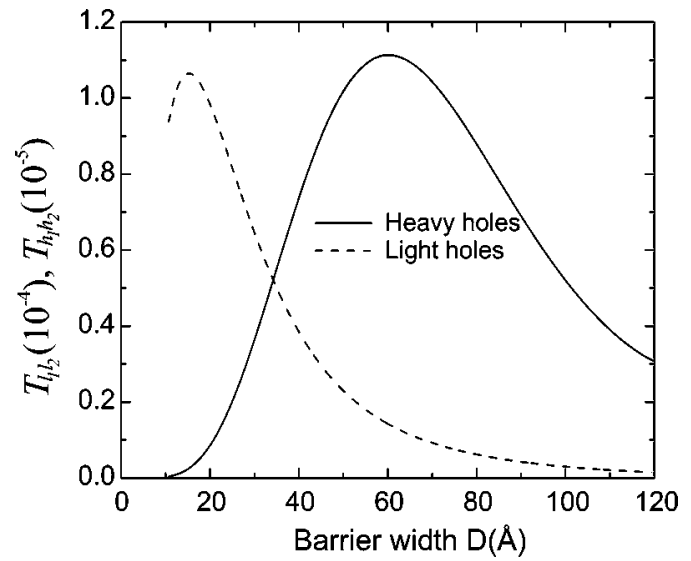

FIG. 7. Transmission coefficient $T_{l_{1} l_{2}}$ between the two light-hole states (solid line) and heavy-hole states $T_{h_{1} h_{2}}$ (dashed line) as a function of barrier width $D$. The magnetic field strength is $B$ $=15 \mathrm{~T}$, and the energy is $E=10 \mathrm{meV}$.

The quantities $k_{x}^{0}, k_{y}^{0}$ are canonical momenta associated with the translational invariance and therefore good quantum numbers throughout the structure. ${ }^{12,22}$ However, within the barrier, where $\mathbf{B} \neq 0$, the third term in the Hamiltonian, Eq. (1), introduces off-diagonal terms due to the matrix $J_{x}$, which represents the projection of the angular momentum on the $x$ axis:

$$
J_{x}=\left(\begin{array}{cccc}
0 & \sqrt{3} i / 2 & 0 & 0 \\
-\sqrt{3} i / 2 & 0 & i & 0 \\
0 & -i & 0 & \sqrt{3} i / 2 \\
0 & 0 & -\sqrt{3} i / 2 & 0
\end{array}\right) \text {. }
$$

Now the transmission between the two light holes (with opposite projection $m= \pm 3 / 2$ ) becomes possible thanks to the presence of the magnetic field. In other words the quantity $m$ is no longer a conserved quantum number since $J_{z}$ does not commute with $J_{x}$ and holes undergo an angular momentum precession. In the inset of Fig. 5(b) the contour plot of the transmission coefficient $T_{h_{1} h_{1}}$ is shown for magnetic field strength $B=15 \mathrm{~T}$. When compared with Fig. 3 we see that the magnetic field enhances the anisotropy. Similar results for $T_{l_{1} l_{1}}$ are shown in the inset of Fig. 5(a). To demonstrate the precession of the hole spin in the barrier we present the transmissions $T_{l_{1} l_{2}}$ and $T_{h_{1} h_{2}}$ as a function of the barrier width $D$ in Fig. 7. Normally the transmission between the states $m=+3 / 2\left(h_{1}\right)$ and $m=-3 / 2\left(h_{2}\right)$ is not possible at $k_{t}=0$ due to zeros in the corresponding terms in the Hamiltonian, Eq. (1). However, the mixing of the heavy holes with the light holes lifts this selection rule ${ }^{23}$ at finite $k_{t}$. This can also be seen if one evaluates $m=\left\langle J_{z}\right\rangle=\left\langle\Psi_{h}\left|\hat{J}_{z}\right| \Psi_{h}\right\rangle$ for heavy holes, which turns into $m=V_{h}^{\dagger} J_{z} V_{h}$ for continuum states or $m=\int V_{h}^{\dagger} J_{z} V_{h} e^{-2 \chi_{z} z} d z, k_{z}=i \chi_{z}$, for evanescent states. The expectation value of the angular momentum on the $z$ axis for the heavy-hole axis decreases with $k_{t}$ below the nominal value of $3 / 2$. This effect was already pointed out for bound states in quantum wells. ${ }^{24}$ Thus one cannot speak about pure 
heavy holes or light holes away from the center of the Brillouin zone. Since the tunneling $T_{h_{1} h_{2}}$ is not a direct effect but is induced by band mixing, its value is smaller than that of $T_{l_{1} l_{2}}$, as can be seen from Fig. 7 (notice there is a factor of 10 difference in the used scale for $T_{l_{1} l_{2}}$ and $T_{h_{1} h_{2}}$ ).

\section{Accuracy of the numerical methods}

This subsection is concerned with the accuracy of the method used here and the accuracy of the MQTBM mentioned earlier, when different numerical implementations of matching conditions at interfaces are used in the latter. It is, in principle, possible to derive analytical expressions for the transmission and reflection coefficients for the problem of a single barrier, but their forms are cumbersome, and we restrict ourselves to the final results. For the case of the method used in this paper, we find that the average relative error obtained is $\delta_{\max }=0.15 \%$, when using $N_{m p}=20000$ mesh points. Now we turn our attention to the MQTBM, where the system of differential equations is approximated as finite difference equations for each mesh point:

$$
\mathbf{H}_{\sigma, \sigma+1} F_{\sigma+1}+\mathbf{H}_{\sigma, \sigma} F_{\sigma}+\mathbf{H}_{\sigma, \sigma-1} F_{\sigma-1}=0,
$$

with $\mathbf{H}_{\sigma \mu}$ being $4 \times 4$ numerical matrices. The boundary conditions on the interface proposed in Ref. 10 read

$$
\begin{gathered}
\mathbf{H}_{\eta, \eta}=\frac{2\left(\mathbf{H}_{R}^{(2)}+\mathbf{H}_{L}^{(2)}\right)}{h^{2}}-i \frac{\mathbf{H}_{R}^{(1)}-\mathbf{H}_{L}^{(1)}}{2 h}, \\
\mathbf{H}_{\eta, \eta+1}=-\frac{2 \mathbf{H}_{R}^{(2)}}{h^{2}}-i \frac{\mathbf{H}_{R}^{(1)}}{2 h}, \\
\mathbf{H}_{\eta, \eta-1}=-\frac{2 \mathbf{H}_{L}^{(2)}}{h^{2}}+i \frac{\mathbf{H}_{L}^{(1)}}{2 h} .
\end{gathered}
$$

These forms of matrices are obtained the conditions $J_{z} F_{L}$ $=J_{z} F_{R}$ is imposed on the wave vectors $F_{\sigma}$. Though mathematically not inconsistent, we find that a direct implementation of the continuity of wave function in our numerical calculations is not appropiate. For instance, if one puts intentionally $\mathbf{H}_{R}^{(i)}=\mathbf{H}_{L}^{(i)}$ (as if there were no changes of materials at the interface), then Eqs. (18a)-(18c) will not reduce to the layer element of a single material [see Eqs. (21a)-(21c)] in Ref. 10). Thus we propose the following forms for $\mathbf{H}_{\sigma \mu}$ :

$$
\begin{gathered}
\mathbf{H}_{\eta, \eta}=\frac{\left(\mathbf{H}_{R}^{(2)}+\mathbf{H}_{L}^{(2)}\right)}{2 h^{2}}+\frac{1}{2}\left(\mathbf{H}_{R}^{(0)}+\mathbf{H}_{L}^{(0)}\right)+V_{\sigma}-E, \\
\mathbf{H}_{\eta, \eta+1}=-\frac{\mathbf{H}_{R}^{(2)}}{h^{2}}-i \frac{\mathbf{H}_{R}^{(1)}}{2 h}, \\
\mathbf{H}_{\eta, \eta-1}=-\frac{\mathbf{H}_{L}^{(2)}}{h^{2}}+i \frac{\mathbf{H}_{L}^{(1)}}{2 h} .
\end{gathered}
$$

When compared with analytical values for $T_{h_{1} h_{1}}$, the new matching relations (19a)-(19c) yield an average relative error $\delta_{a v 1}<0.2 \%$ for $N_{m p}=300$, while the "old" relations (19a)-(19c) give $\delta_{a v 2}=3 \%$ for the same $N_{m p}$ which is an improvement with more than a factor 10 .

\section{RESONANT TUNNELING}

When a bias is applied across the structure there is a net current flowing from the left to the right lead, if there are unoccupied states on the right lead. Taking into account $\left|t_{\lambda \mu}^{0}\right|^{2} v_{z \mu}^{(R)}=T_{\lambda \mu} v_{z \lambda}^{(L)}$ the expression for the current density is

$$
\begin{aligned}
J_{C}= & \frac{e}{8 \pi^{3}} \int d k_{x} d k_{y} d k_{z}\left\{\sum_{\mu} T_{h_{1} \mu} v_{z h_{1}}^{(L)}+\sum_{\mu} T_{h_{2} \mu} v_{z h_{2}}^{(L)}\right. \\
& \left.+\sum_{\mu} T_{h_{1} \mu} v_{z l_{1}}^{(L)}+\sum_{\mu} T_{l_{2} \mu} v_{z l_{2}}^{(L)}\right\}\left[f(E)-f\left(E+e V_{b}\right)\right] .
\end{aligned}
$$

However, since the transmission coefficients are calculated at fixed $E$, it is better to change the variable $k_{z}$ into $E_{z}$, which leads to the following expression for the current:

$$
\begin{aligned}
J_{C}= & \frac{e}{8 \pi^{3} \hbar} \int_{0}^{k_{t \max }} k_{t} d k_{t} \int_{0}^{2 \pi} d \phi d E\left(\sum_{\mu \lambda} T_{\mu \lambda}\right) \\
& \times\left[f(E)-f\left(E+e V_{b}\right)\right] .
\end{aligned}
$$

where it is assumed that $d E=d E_{z}$ since integration over $k_{t}$ is in the front of integral over $d E_{z}$. The tunneling current is calculated for a double-barrier resonant structure (DBRT). However, another set of values of the Luttinger parameters is taken, $\gamma_{1}=7.65, \gamma_{2}=2.845, \gamma_{3}=2.845, \kappa=1.72$ (for both materials), in order to make a comparison with the results of Ref. 13 possible. The other parameters are taken from the same reference: barrier height $V_{0}=0.1 \mathrm{eV}$, barrier width $D$ $=25 \AA$, well width $W=80 \AA$, and Fermi energy $E_{F}$ $=10 \mathrm{meV}$. The current density as a function of applied bias is plotted in Fig. 8, along with results of Ref. 13 (dashed line) for $T=0 \mathrm{~K}$. The first two peaks correspond (neglecting the small one, $\mathrm{HHO}$, at small $V_{\text {bias }}$ ) to the first light-hole resonance (in the well) LHO and the second heavy-hole resonance HH1. In Ref. 13 the explicit dependence of the current on the in-plane momenta was neglected in order to facilitate numerical integration in Eq. (21). Though the two curves are of the same order of magnitude, the two peak structure is not reproduced with the approximation $J_{C}\left(E, k_{\|}\right) \approx J_{C}\left(E_{z}\right)$. These two peaks indeed coincide with one another at $k_{t}=0$, but because of the different mass of the heavy and light holes and, more importantly, because of band mixing, the position of the resonant levels in the well between the two barriers split at $k_{t}>0$.

Now we revert to the previous values for the Luttinger parameters: $\gamma_{1}^{a}=6.85, \gamma_{2}^{a}=2.1$, etc. (see previous section), but the same dimensions of the DBRT-i.e., $D=25 \AA \mathrm{W}=80 \AA$. The transmission coefficients $T_{h_{1} \lambda}$ and $T_{l_{\lambda} \lambda} \quad(\lambda$ $\left.\in\left\{h_{1}, h_{2}, l_{1}, l_{2}\right\}\right)$ when an external magnetic field $\mathbf{B}=10 \mathrm{~T}$ is 


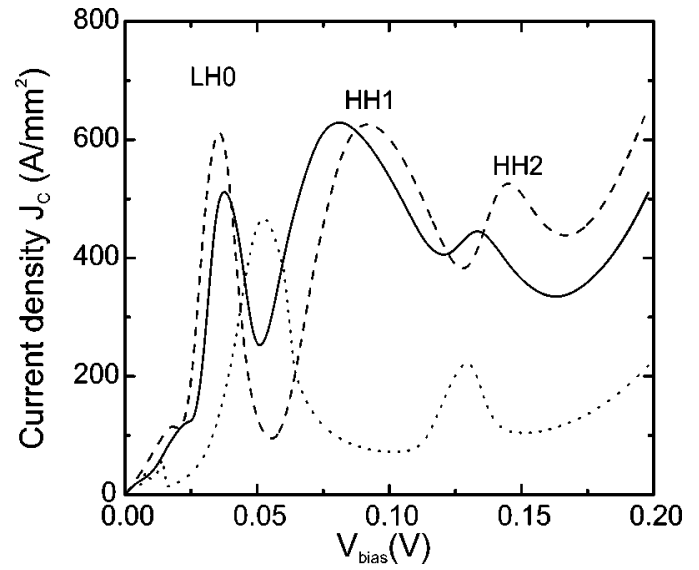

FIG. 8. Tunneling current through DBRT as a function of applied bias at $\mathbf{B}=0$ in arbitrary units (solid line) in the spherical approximation, $\gamma_{2}=\gamma_{3}$. As a reference $J_{C}\left(V_{\text {bias }}\right)$ is shown (dotted line) when the approximation $k_{t}=0$ is used as in Ref. 13. The dashed line represents the values of the current calculated without approximations.

applied in plane (except in the contact regions) -i.e., parallel to the layers - are shown in Figs. 9(a) and 9(b), respectively. Out of 16 possible transmission channels, only 8 are different and shown in the figures, due to the fact that the magnetic field is normal to the quantization axis and there is no bias.

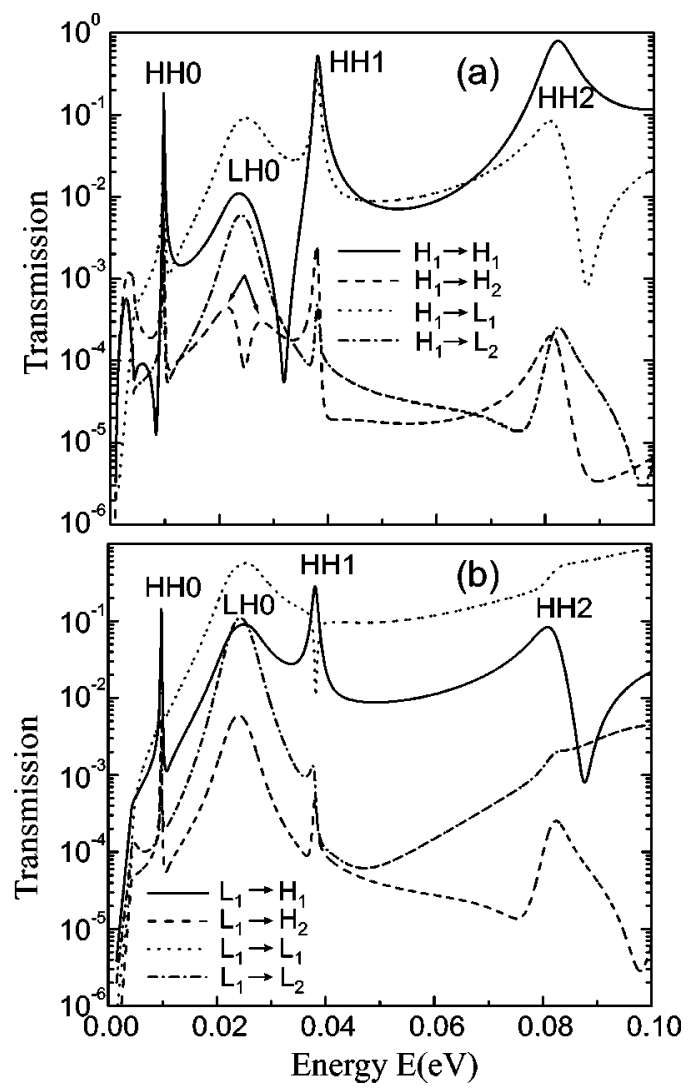

FIG. 9. Transmission coefficients of: (a) the heavy hole $h_{1}$ and (b) the light hole $l_{1}$ to other four possible hole states as a function of the energy of the incoming hole, in the presence of an in-plane magnetic field $B=10 \mathrm{~T}$.

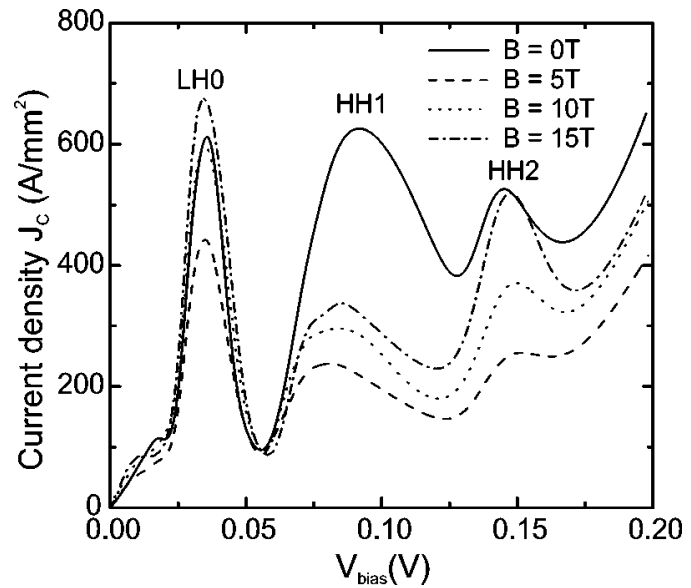

FIG. 10. Tunneling current through DBRT as a function of applied bias for $B=0 \mathrm{~T}$ (solid line), $B=5 \mathrm{~T}$ (dashed line), $B=10 \mathrm{~T}$ (dotted line), and $B=15 \mathrm{~T}$ (dash-dotted line).

The peaks in $T_{\lambda \mu}$ that correspond to the light-hole resonance LHO are broader than those that correspond to HH resonances since the light holes reside shorter in the structure. It can be seen that the $\mathrm{HH}_{x}$ resonant peaks do not show any visible splitting when a magnetic field is applied along the $x$ axis. This is a consequence of the fact that the quantization axis for angular momentum is chosen to be along $z$ and the operator $\hat{S}_{z}$ for the heavy-hole states at $k_{t}=0$ has definite values $s_{z}= \pm 1$. Thus expectation value of $\hat{J}_{x}$ between the states of the heavy holes at $k_{t}=0$ is zero ${ }^{13,25}$-i.e., $\left\langle J_{x}\right\rangle=0$. This conclusion becomes a little bit more relaxed for larger in-plane momenta, but still the heavy-hole splitting is significantly smaller than that of the light holes.

Finally, the current density is given in Fig. 10 for four different values of the magnetic field strength at $T=0 \mathrm{~K}$. The amplitudes of the peaks in general decrease with an increase of the magnetic field, as the vector potential component $e A_{y} / \hbar$ narrows the range of $k_{y}^{0}$ in momentum space that can ensure efficient tunneling. However, we observe that the increase of the current amplitude appears around $B \approx 12 \mathrm{~T}$. The nonmonotonic dependence of the peak current in inplane fields was observed ${ }^{26}$ in magnetotunneling of electrons in DBRT, so that it is not directly related to band mixing. Though we may expect a splitting to appear in LHO, it eventually does not. The absence of spin splitting (even for LH0) in DBRT under an in-plane magnetic field was already noted experimentally ${ }^{27}$ and theoretically. ${ }^{13}$ This is probably due to the integration over both $E$ and $k_{t}$ which smears out the spinsplit peaks into a single peak. The heavy-hole component of the current is shown in Fig. 11. It is apparent that the peak LH0 increases with $B$ as the heavy holes acquire light-hole character, thus lowering their effective mass. To check spin polarization effects in DBRT one needs to evaluate the ratio

$$
P=\left(J_{C}^{\uparrow}-J_{C}^{\downarrow}\right) / J_{C}=\Delta J_{C} / J_{C},
$$

where 


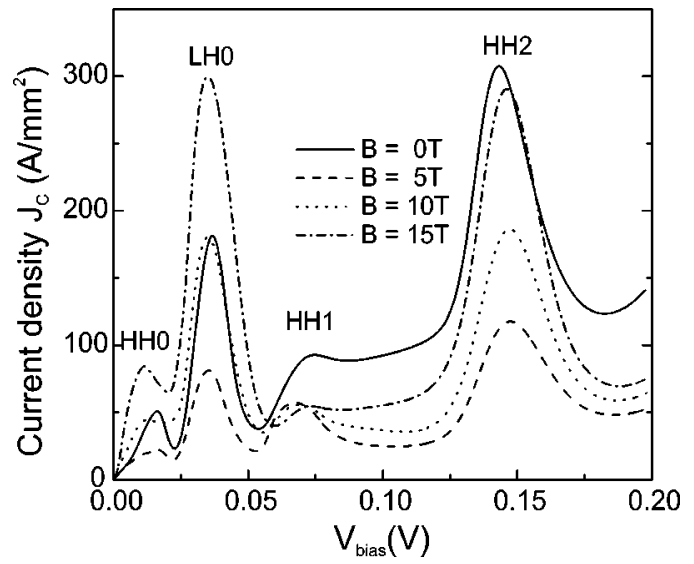

FIG. 11. Heavy-hole current component as a function of applied bias for $B=0 \mathrm{~T}$ (solid line), $B=5 \mathrm{~T}$ (dashed line), $B=10 \mathrm{~T}$ (dotted line), and $B=15 \mathrm{~T}$ (dash-dotted line).

$$
\begin{aligned}
\Delta J_{C}= & \frac{e}{8 \pi^{3} \hbar} \int k_{t} d k_{t} d \phi d E\left(\sum_{\mu \lambda} T_{\mu \lambda}\left\langle V_{\lambda}\left|\hat{\sigma}_{z}\right| V_{\lambda}\right\rangle\right) \\
& \times\left[f(E)-f\left(E+e V_{b}\right)\right] .
\end{aligned}
$$

Here $\hat{\sigma}_{z}$ is the Pauli spin operator acting as a weighting factor in the output channels in Eq. (22) and reads

$$
\sigma_{z}=\left(\begin{array}{ccccccc}
1 & 0 & 0 & 0 & \cdot & 0 & 0 \\
0 & \frac{1}{3} & 0 & 0 & \cdot & \frac{2 \sqrt{2} i}{3} & 0 \\
0 & 0 & -\frac{1}{3} & 0 & \cdot & 0 & -\frac{2 \sqrt{2} i}{3} \\
0 & 0 & 0 & -1 & . & 0 & 0 \\
. . & . \cdot & . . & . . & . & & \\
0 & -\frac{2 \sqrt{2} i}{3} & 0 & 0 & -\frac{1}{3} & 0 \\
0 & 0 & \frac{2 \sqrt{2} i}{3} & 0 & 0 & \frac{1}{3}
\end{array}\right) .
$$

For four-component vectors (neglecting the SO band) this operator is actually equal to $\frac{2}{3} J_{z}$ [marked by the dotted line in Eq. (22)] in the subspace of heavy and light holes $\left|3 / 2, j_{z}\right\rangle$, as was already pointed out during the derivation of the hole Zeeman term in Ref. 5. In the limit of a strong spin-orbit coupling and a small Fermi energy $E_{F} / \Delta \rightarrow 0$, as in our case, the $4 \times 4 \mathrm{LK}$ is a good approximation ${ }^{28}$ to describe the holes. The spin polarization of the current and its HH and HL components as a function of magnetic field are given in Fig. 12, for an applied bias $V_{\text {bias }}=0.1 \mathrm{eV}$. The currents are slightly polarized even at $B=0$, which is a consequence of the lifting of the Kramers degeneracy due to structural inversion asymmetry (SIA). The polarization exhibits oscillating behavior with the magnetic field strength, though its amplitude in general increases with $B$. The maximal polarization that can be

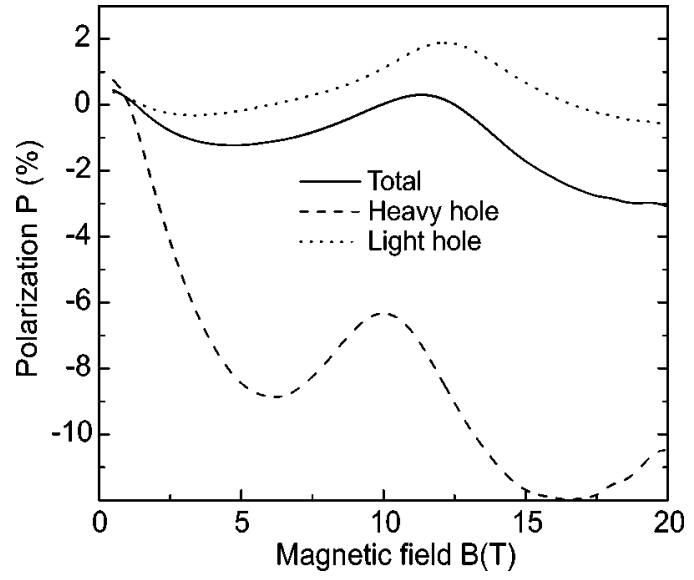

FIG. 12. Spin polarization $P$ of the current as a function of in-plane magnetic field for bias $V_{\text {bias }}=0.1 \mathrm{eV}$ : total (solid line), heavy-hole (dotted line), and light-hole (dashed line) polarization.

achieved for the present dimensions DBRT is around 3\%. The spin polarization can be enhanced by tuning the external bias $V_{\text {bias }}$ or/and well width $W$. We note, however, at this point that $P$ is not a monotonous function of $V_{\text {bias }}$ and $W$.

\section{CONCLUSIONS}

In this paper the tunneling of holes is investigated within the frame of the $4 \times 4$ Luttinger-Kohn Hamiltonian in the most general case where $\gamma_{1} \neq \gamma_{2} \neq \gamma_{3}$. The transmission coefficients are plotted as a function of in-plane momenta $\left(k_{x}, k_{y}\right)$, which is a conserved quantum number throughout the structure. The conservation of probability current density-i.e., the sum of all transmission and reflection coefficients equals unity-is confirmed to be valid in all cases, contrary to the conclusions of Ref. 18. The anisotropy caused by the difference in the Luttinger parameters in $\gamma_{2} \neq \gamma_{3}$ is reflected mainly in the transmission between the two heavy holes (with or without external magnetic field), because their propagating states exist for larger $\left(k_{x}, k_{y}\right)$ (than that of the light hole) when band mixing plays an important role. The range of parameters $\left(E, k_{x}, k_{y}\right)$ for case (iii), where the light holes propagate at group velocity opposite to their phase velocity, is narrow and transmission coefficients are small, so its influence on the current is not significant. However, we find that employing any approximation, such as spherical, isotropical, or neglecting the dependence of $J_{C}$ on $\left(k_{x}, k_{y}\right)$, can lead to visible discrepancies as was shown in Fig. 8. The main consequence of band mixing is that heavy holes acquire light-hole character in the barrier, thus enhancing their tunneling. The values of $T_{h_{1} h_{1}}$ are larger than in the one-band picture and increase with $\left(k_{x}, k_{y}\right)$ as long as both heavy and light holes exist in the external leads [case (i)]. In the presence of an in-plane magnetic field (normal to the current) the holes acquire an additional in-plane momentum $k_{y}$ and for certain values of $\left(E, k_{x}, k_{y}\right)$ heavy holes stay longer in the barrier, thus enhancing their (partial) light-hole character. Then transmission between the two light holes is possible; i.e., the hole undergoes a precession. The heavy holes at $k_{t}$ 
$=0$ cannot precess because of the zeros in the Hamiltonian between $h_{1}$ and $h_{2}$. However, the band mixing lifts this "selection rule" as heavy holes acquire some of the light-hole character. We presented results for the current density of DBRT which is an interesting structure for possible applications, and it is a common setup in experiments. Upon comparing results with other theoretical results already present in the literature, we find that band mixing is important and cannot be neglected. At the end, we presented the ratio of spin polarization $P$ as a function of magnetic field. Spin polariza- tion can be enhanced by tuning the well width and/or by an increase of applied bias.

\section{ACKNOWLEDGMENTS}

This work was supported by the Flemish Science Foundation (FWO-V1), the Belgian Science Policy, and the "Onderzoeksraad van de Universiteit Antwerpen" (GOA). P.K. is grateful to Professor Milan Tadić for useful suggestions.
*Electronic address: predrag.krstajic@ua.ac.be

†Electronic address: francois.peeters@ua.ac.be

${ }^{1}$ H. Ohno, H. Munekata, T. Penny, S. von Molnar, and L. L. Chang, Phys. Rev. Lett. 68, 2664 (1992).

${ }^{2}$ M. Tanaka and Y. Higo, Phys. Rev. Lett. 87, 026602 (2001).

${ }^{3}$ H. Ohno, F. Matsukura, and Y. Ohno, Solid State Commun. 119, 281 (2001).

${ }^{4}$ J. M. Luttinger and W. Kohn, Phys. Rev. 97, 869 (1955).

${ }^{5}$ J. M. Luttinger, Phys. Rev. 102, 1030 (1956).

${ }^{6}$ I. Vurgaftman and J. R. Meyer, Phys. Rev. B 67, 125209 (2003).

${ }^{7}$ R. Wessel and M. Altarelli, Phys. Rev. B 39, 12802 (1989).

${ }^{8}$ E. T. Yu, M. K. Jackson, and T. C. McGill, Appl. Phys. Lett. 55, 744 (1989).

${ }^{9}$ J.-B. Xia, Phys. Rev. B 38, 8365 (1998).

${ }^{10}$ D. Z.-Y. Ting, E. T. Yu, and T. C. McGill, Phys. Rev. B 45, 3583 (1992).

${ }^{11}$ Y. X. Liu, D. Z.-Y. Ting, and T. C. McGill, Phys. Rev. B 54, 5675 (1996).

${ }^{12}$ G. Y. Wu, K.-M. Hung, and C.-J. Chen, Phys. Rev. B 46, 1521 (1992).

${ }^{13}$ T.-J. Chow, G. Y. Wu, K.-M. Hung, and C.-W. Chen, Phys. Rev. B 55, 1329 (1997).

${ }^{14}$ J.-X. Zhu, Z. D. Wang, and C.-D. Gong, Solid State Commun. 101, 257 (1997).

${ }^{15}$ A. C. Rodrigues Bittencourt, A. M. Cohen, and G. E. Marques,
Phys. Rev. B 57, 4525 (1998).

${ }^{16} \mathrm{G}$. Bastard, Wave Mechanics Applied to Semiconductor Heterostructures (Les Editions de Physique, Paris, 1987), p. 45.

${ }^{17}$ L. C. Andreani, A. Pasquarello, and F. Bassani, Phys. Rev. B 36, 5887 (1987).

${ }^{18}$ C. Y. Chao and S. L. Chuang, Phys. Rev. B 43, 7027 (1991).

${ }^{19}$ W. R. Frensley, Rev. Mod. Phys. 62, 745 (1990).

${ }^{20}$ F. Sheid, Numerical Analysis, Shaum's Outline Series (McGrawHill, New York, 1968), p. 194.

${ }^{21}$ M. K. Jackson, M. B. Johnson, D. H. Chow, T. C. McGill, and C. W. Nieh, Appl. Phys. Lett. 54, 552 (1989).

${ }^{22}$ L. D. Landau and E. M. Lifshitz, Quantum Mechanics (Pergamon Press, Oxford, 1977), p. 456.

${ }^{23}$ A. G. Petukhov, D. O. Demchenko, and A. N. Chantis, Phys. Rev. B 68, 125332 (2003); A. G. Petukhov, W. R. L. Lambrecht, and B. Segall, ibid. 53, 3646 (1996).

${ }^{24}$ R. Ferreira and G. Bastard, Phys. Rev. B 43, 9687 (1991).

${ }^{25}$ G. Goldoni and A. Fasolino, Phys. Rev. B 48, 4948 (1993).

${ }^{26}$ A. Zaslavsky, Y. P. Li, C. Tsui, M. Santos, and M. Shayegan, Phys. Rev. B 42, 1374 (1990).

${ }^{27}$ R. K. Hayden, D. K. Maude, L. Eaves, E. C. Valadares, M. Henini, F. W. Sheard, O. H. Hughes, J. C. Portal, and L. Cury, Phys. Rev. Lett. 66, 1749 (1991).

${ }^{28}$ G. Edwards, E. C. Valadares, and F. W. Sheard, Phys. Rev. B 50, 8493 (1994). 